\title{
Exponential Stability of the Monotubular Heat Exchanger Equation with Time Delay in Boundary Observation
}

\author{
Xue-Lian Jin, ${ }^{1}$ Yang Zhang, ${ }^{2}$ Fu Zheng, ${ }^{2}$ and Bao-zhu Guo ${ }^{3}$ \\ ${ }^{1}$ Science College, Liaoning University of Technology, Jinzhou 121001, China \\ ${ }^{2}$ Department of Mathematics, Bohai University, Jinzhou 121013, China \\ ${ }^{3}$ Academy of Mathematics and Systems Science, CAS, Beijing 100190, China \\ Correspondence should be addressed to Fu Zheng; zhengfu@amss.ac.cn
}

Received 7 February 2017; Accepted 17 May 2017; Published 20 June 2017

Academic Editor: Cheng Z. Xu

Copyright (C) 2017 Xue-Lian Jin et al. This is an open access article distributed under the Creative Commons Attribution License, which permits unrestricted use, distribution, and reproduction in any medium, provided the original work is properly cited.

\begin{abstract}
The exponential stability of the monotubular heat exchanger equation with boundary observation possessing a time delay and inner control was investigated. Firstly, the close-loop system was translated into an abstract Cauchy problem in the suitable state space. A uniformly bounded $C_{0}$-semigroup generated by the close-loop system, which implies that the unique solution of the system exists, was shown. Secondly, the spectrum configuration of the closed-loop system was analyzed and the eventual differentiability and the eventual compactness of the semigroup were shown by the resolvent estimates on some resolvent sets. This implies that the spectrum-determined growth assumption holds. Finally, a sufficient condition, which is related to the physical parameters in the system and is independent of the time delay, of the exponential stability of the closed-loop system was given.
\end{abstract}

\section{Introduction}

In the past decades, the monotubular heat exchanger system has been analyzed by several researchers from the viewpoint of system theory. For example, $\mathrm{Xu}$ et al. [1] have treated the heat exchanger equation with zero boundary conditions and proved the exponential stability for each set of physical parameters with the finest estimate of the decay rate by using Huang's result [2] on the spectrum-determined growth assumption (see also [3]). In [4], Kanoh has introduced a controller with two kinds of feedback loops for the monotubular heat exchanger equation. However, he does not discuss the exponential stability of the closed-loop system, which is one of the important properties in the field of dynamical system theory. In [5], Sano analyzes the exponential stability of the monotubular heat exchanger system with static output feedback. In [6], Guo and Liang show that the $C_{0}$-semigroup associated with the closed-loop system of the monotubular heat exchanger equation is differentiable after some finite time period. Moreover, they verify that the system is not a Riesz spectral system although the root subspace is complete in the energy Hilbert space. Meanwhile, Yu and Liu give the differentiability of the system with static output feedback by the different method in [7].

However, in a practical control system, there is often a time delay between the controller to be implemented and the information via the observation of the system. These hereditary effects are sometimes unavoidable because they might turn a well-behaved system into a wild one. A simple example can be found in the work of Gumowski and Mira [8], where they demonstrated that the occurrence of delays could destroy the stability and cause periodic oscillations in a system governed by differential equation. Another example from Datko [9] illustrated that an arbitrary small time delay in the control could destabilize a boundary feedback hyperbolic control system. On the other side, the inclusion of an appropriate time delay effect can sometimes improve the performance of the system (e.g., see $[10,11]$ ). The stabilization with time delay in observation or control represents difficult mathematical challenges in the control of distributed parameter systems. However, this does not mean that there is no stabilizing controller in the presence of time delay. You can refer to [12-18] for some successful examples. 
Motivated by these works, we shall introduce time delay to the monotubular heat exchanger system and we then investigate the effect of the time delay on exponential stability of the system. More precisely, we assume that a time delay occurs in the boundary observation. We want to pose a question. Is the stabilization robust to the time delay for the proportional feedback controller? The present paper is devoted to answering this question.

The content of this paper is organized as follows. In Section 2, we shall introduce the monotubular heat exchanger system mentioned above and formulate our problem in a suitable Hilbert space. We show that the closed-loop system generates a $C_{0}$-semigroup of bounded linear operators and obtain the well-posedness of the system. In Section 3, we carry out detailed spectral analysis and obtain the spectrum configuration of the closed-loop system. Furthermore, we present that $C_{0}$-semigroup is differentiable and compact after some finite time period based on the spectral analysis. This means that the spectrum-determined growth assumption holds. In Section 4, the stable regions of the closed-loop system are given by verifying that the spectral bound is less than or greater than zero. In the last section, a concise conclusion is given.

\section{System Description and Well-Posedness of the System}

We shall consider the following type of monotubular heat exchanger equation in which the time delay occurs in boundary observation:

$$
\begin{aligned}
\frac{\partial z}{\partial t}(t, x) & =-\frac{\partial z}{\partial x}(t, x)-a z(t, x)+\gamma e^{-b x} u(t) \\
\qquad(t, x) \in(0, \infty) \times(0,1) & \\
z(t, 0) & =0 \\
z(0, x) & =z_{0}(x) \\
y(t) & =z(t-\tau, 1)
\end{aligned}
$$

where $z(t, x) \in \mathbb{R}$ is the temperature variation at the time $t$ and at the point $x \in[0,1]$ with respect to an equilibrium point, $u(t) \in \mathbb{R}$ is the control input, $y(t) \in \mathbb{R}$ is the measured output, $a$ is a positive physical parameter, and $\gamma e^{-b x}$ denotes the spatial distribution of an actuator, with $b$ and $\gamma$ being positive constants, and $\tau>0$ is the length of time delay.

As usual, we adopt the simple feedback control law $u(t)=$ $-k y(t)$ in system (1) which results in the following closedloop system:

$$
\begin{aligned}
\frac{\partial z}{\partial t}(t, x) & =-\frac{\partial z}{\partial x}(t, x)-a z(t, x)-k \gamma e^{-b x} z(t-\tau, 1) \\
(t, x) \in(0, \infty) \times(0,1), & \\
z(t, 0) & =0, \\
z(0, x) & =z_{0}(x) .
\end{aligned}
$$

Setting $w(t, x)=z(t-x \tau, 1)$, system (2) is equivalent to

$$
\begin{aligned}
\frac{\partial z}{\partial t}(t, x) & =-\frac{\partial z}{\partial x}(t, x)-a z(t, x)-k \gamma e^{-b x} w(t, 1), \\
\tau \frac{\partial w}{\partial t}(t, x) & =-\frac{\partial w}{\partial x}(t, x), \quad \\
z(t, 0) & =0 \\
z(t, 1) & =w(t, 0), \\
z(0, x) & =z_{0}(x), \quad t \in(0, \infty), \\
w(0, x) & =f(-\tau x),
\end{aligned}
$$

$$
x \in(0,1),
$$

in which the function $f(\cdot)$ denotes the history of the system at the boundary $x=1$ at the time period $(-\tau, 0)$.

We take the state Hilbert space $\mathscr{H}=L^{2}[0,1] \times L^{2}[0,1]$ equipped with natural inner product $\langle\cdot, \cdot\rangle$ and the induced norm $\|\cdot\|$. Define the operator $A: D(A) \subset \mathscr{H} \rightarrow \mathscr{H}$ as

$$
\begin{aligned}
& A\left(\begin{array}{l}
z \\
w
\end{array}\right)=\left(\begin{array}{c}
-z^{\prime}(x)-a z(x)-k \gamma e^{-b x} w(1) \\
\frac{-1}{\tau} w^{\prime}(x)
\end{array}\right), \\
& (z, w)^{\top} \in D(A), \\
& D(A)=\left\{(z, w) \in H^{1}(0,1) \times H^{1}(0,1) \mid z(0)\right. \\
& \quad=0, z(1)=w(0)\} .
\end{aligned}
$$

Thus, system (3) can be written as

$$
\begin{aligned}
& \frac{d}{d t} X(t)=A X(t) \\
& X(t)=\left(\begin{array}{c}
z(t, x) \\
w(t, x)
\end{array}\right) \in \mathscr{H} \forall t \geq 0, X(0)=\left(\begin{array}{c}
z_{0} \\
f
\end{array}\right) .
\end{aligned}
$$

If the operator $A$ generates a $C_{0}$-semigroup $T(t)$ on $\mathscr{H}$, then (5) has a unique solution, which is equivalent to the unique solution to (2) or (3) exists. More precisely, we have the following theorem.

Theorem 1. For any $k \in \mathbb{R}$, the operator $A$ defined by (4) generates a $C_{0}$-semigroup on $\mathscr{H}$.

Proof. Firstly, in order to prove that $A$ generates a $C_{0}$ semigroup, we introduce a new equivalent inner product in $\mathscr{H}$ :

$$
\begin{aligned}
\left\langle X_{1}, X_{2}\right\rangle_{\tau}= & \int_{0}^{1} z_{1}(x) \overline{z_{2}(x)} d x \\
& +\tau \int_{0}^{1} w_{1}(x) \overline{w_{2}(x)} d x \\
& X_{i}=\left(z_{i}, w_{i}\right)^{\top} \in \mathscr{H}(i=1,2) .
\end{aligned}
$$


From the definition of the operator $A$ in (4), it follows that the identities

$$
\begin{aligned}
2 \operatorname{Re} & \left\langle A\left(\begin{array}{c}
z \\
w
\end{array}\right),\left(\begin{array}{c}
z \\
w
\end{array}\right)\right\rangle_{\tau} \\
= & -|w(1)|^{2}-2 a \int_{0}^{1}|z(x)|^{2} d x \\
& -\int_{0}^{1} 2 k \gamma e^{-b x} \operatorname{Re}[w(1) \overline{z(x)}] d x \\
\leq & -|w(1)|^{2}-2 a \int_{0}^{1}|z(x)|^{2} d x \\
& -\int_{0}^{1} 2 k \gamma e^{-b x} \operatorname{Re}[w(1) \overline{z(x)}] d x \\
\leq & -|w(1)|^{2}-2 a \int_{0}^{1}|z(x)|^{2} d x \\
& +\int_{0}^{1}|w(1)|^{2}+k^{2} \gamma^{2}|z(x)|^{2} d x \\
= & \left(k^{2} \gamma^{2}-2 a\right) \int_{0}^{1}|z(x)|^{2} d x
\end{aligned}
$$

hold. These identities imply that

$$
\operatorname{Re}\left\langle A\left(\begin{array}{l}
z \\
w
\end{array}\right),\left(\begin{array}{l}
z \\
w
\end{array}\right)\right\rangle_{\tau} \leq\left(\frac{k^{2} \gamma^{2}}{2}-a\right)\left\|\left(\begin{array}{l}
z \\
w
\end{array}\right)\right\|_{\tau},
$$

in which $\|\cdot\|_{\tau}$ denotes the norm induced by the inner product of $\langle\cdot, \cdot\rangle_{\tau}$ in $\mathscr{H}$.

Secondly, it is easy to verify that the operator $A$ is densely defined and closed and its adjoint with respect to the new inner product is given as follows:

$$
\begin{aligned}
& A^{*}\left(\begin{array}{l}
z \\
w
\end{array}\right)=\left(\begin{array}{c}
z^{\prime}(x)-a z(x) \\
\frac{1}{\tau} w^{\prime}(x)
\end{array}\right), \quad(z, w)^{\top} \in D\left(A^{*}\right), \\
& D\left(A^{*}\right)=\left\{(z, w) \in H^{1}(0,1) \times H^{1}(0,1) \mid w(0)\right. \\
& \left.=z(1), w(1)=-k \gamma \int_{0}^{1} e^{-b x} z(x) d x\right\} .
\end{aligned}
$$

By the same method as above, we have that, for all $(z, w)^{\top} \epsilon$ $D\left(A^{*}\right)$,

$$
\begin{aligned}
\operatorname{Re}\left\langle A^{*}\left(\begin{array}{l}
z \\
w
\end{array}\right),\left(\begin{array}{l}
z \\
w
\end{array}\right)\right\rangle_{\tau} \\
=-\left(\frac{1}{2}\right)|z(0)|^{2}-a \int_{0}^{1}|z(x)|^{2} d x \\
+\frac{1}{2}\left|k \gamma \int_{0}^{1} e^{-b x} z(x) d x\right|^{2} \\
\leq\left(\frac{k^{2} \gamma^{2}}{2}-a\right)\left\|\left(\begin{array}{c}
z \\
w
\end{array}\right)\right\|_{\tau} .
\end{aligned}
$$

Finally, together with (8) and (10), it follows that the operator $A$ generates a $C_{0}$-semigroup $T(t)$ on $\left(\mathscr{H},\|\cdot\|_{\tau}\right)$ satisfying $\|T(t)\|_{\tau} \leq e^{\left(k^{2} \gamma^{2} / 2-a\right) t}$ by using Corollary 2.2 .3 of [19]. The equivalence of two norms $\|\cdot\|_{\tau}$ and $\|\cdot\|$ on $\mathscr{H}$ implies that there exists a positive constant $M$ such that $\|T(t)\| \leq$ $M e^{\left(k^{2} \gamma^{2} / 2-a\right) t}$. Thus, the proof of the theorem is complete.

\section{Spectral Analysis and Regularity of Semigroup}

In order to show the exponential stability of system (5), we shall analyze the spectral configuration of the operator $A$. To this end, we show that the operator $A$ is of compact resolvent (in other words, $A$ is a discrete operator).

Theorem 2. We have the following statements:

(1) $\lambda \in \rho(A)$ if and only if $\lambda$ satisfies

$$
-k \gamma e^{-\lambda \tau} e^{-(\lambda+a)} \int_{0}^{1} e^{(\lambda+a-b) s} d s \neq 1 .
$$

(2) If $\lambda \in \rho(A)$, then $R(\lambda, A)$ is compact.

Proof. (1) For arbitrary $(h, g)^{\top} \in \mathscr{H}$, let us consider the resolvent equation

$$
(\lambda I-A)\left(\begin{array}{l}
z \\
w
\end{array}\right)=\left(\begin{array}{l}
h \\
g
\end{array}\right) .
$$

It is equivalent to

$$
\begin{aligned}
z^{\prime}(x) & =-(\lambda+a) z(x)-k \gamma e^{-b x} w(1)+h(x), \\
w^{\prime}(x) & =-\lambda \tau w(x)+\tau g(x), \\
z(0) & =0, \\
z(1) & =w(0) .
\end{aligned}
$$

Solving the equations of (13) and (14) with the help of $z(0)=$ 0 , we have

$$
\begin{aligned}
& z(x)=\int_{0}^{x} e^{-(\lambda+a)(x-s)}\left[-k \gamma e^{-b s} w(1)+h(s)\right] d s \\
& w(x)=w(0) e^{-\lambda \tau x}+\tau \int_{0}^{x} e^{-\lambda \tau(x-s)} g(s) d s .
\end{aligned}
$$

It follows from $z(1)=w(0)$ that

$$
\begin{aligned}
& w(0)+k \gamma e^{-(\lambda+a)} \int_{0}^{1} e^{(\lambda+a-b) s} d s w(1) \\
& =\int_{0}^{1} e^{-(\lambda+a)(1-s)} h(s) d s, \\
& -w(0) e^{-\lambda \tau}+w(1)=\tau \int_{0}^{1} e^{-\lambda \tau(1-s)} g(s) d s .
\end{aligned}
$$

It is easy to see from (16) that $z(x)$ and $w(x)$ are uniquely determined by $w(0)$ and $w(1)$. However, (17) on $w(0)$ and 
$w(1)$ has unique solution if and only if its coefficient determinant is not zero. That is,

$$
\Delta(\lambda):=\left|\begin{array}{cc}
1 & k \gamma e^{-(\lambda+a)} \int_{0}^{1} e^{(\lambda+a-b) s} d s \\
-e^{-\lambda \tau} & 1
\end{array}\right| \neq 0 .
$$

A simple computation shows that statement (1) is right.

(2) If we let $r(\lambda)=-k \gamma e^{-(\lambda+a)} \int_{0}^{1} e^{(\lambda+a-b) s} d s$ and $\lambda \in \rho(A)$, then we have

$$
\begin{gathered}
w(0)=\frac{1}{\Delta(\lambda)}\left[\int_{0}^{1} e^{-(\lambda+a)(1-s)} h(s) d s\right. \\
\left.+\operatorname{\tau r}(\lambda) \int_{0}^{1} e^{-\lambda \tau(1-s)} g(s) d s\right], \\
w(1)=\frac{1}{\Delta(\lambda)}\left[\tau \int_{0}^{1} e^{-\lambda \tau(1-s)} g(s) d s\right. \\
\left.+e^{\lambda \tau} \int_{0}^{1} e^{-(\lambda+a)(1-s)} h(s) d s\right] .
\end{gathered}
$$

It follows from (16) that

$$
\begin{aligned}
& R(\lambda, A)\left(\begin{array}{l}
h(x) \\
g(x)
\end{array}\right) \\
& =\left(\begin{array}{c}
k \gamma w(1) \int_{0}^{x} e^{-(\lambda+a)(x-s)} e^{-b s} d s+\int_{0}^{x} e^{-(\lambda+a)(x-s)} h(s) d s \\
w(0) e^{-\lambda \tau x}+\tau \int_{0}^{x} e^{-\lambda \tau(x-s)} g(s) d s
\end{array}\right) .
\end{aligned}
$$

Set

$$
\begin{aligned}
& T_{1}\left(\begin{array}{l}
h(x) \\
g(x)
\end{array}\right)=\left(\begin{array}{c}
k \gamma w(1) \int_{0}^{x} e^{-(\lambda+a)(x-s)} e^{-b s} d s \\
w(0) e^{-\lambda \tau x}
\end{array}\right), \\
& T_{2}\left(\begin{array}{c}
h(x) \\
g(x)
\end{array}\right)=\left(\begin{array}{l}
\int_{0}^{x} e^{-(\lambda+a)(x-s)} h(s) d s \\
\tau \int_{0}^{x} e^{-\lambda \tau(x-s)} g(s) d s
\end{array}\right) .
\end{aligned}
$$

Obviously, $R(\lambda, A)=T_{1}+T_{2}$ and $T_{1}$ and $T_{2}$ are compact operators on $\mathscr{H}$. These facts imply that $R(\lambda, A)$ is compact. Thus, the second statement holds and the proof of Theorem 2 is completed.

Similarly, we have the following spectral distribution and omit its proof since it involves only simple calculations.

Lemma 3. $\lambda=0$ is an eigenvalue of the operator $A$ if and only if $k \gamma\left(e^{-a}-e^{-b}\right)=a-b \cdot \lambda=-a+b$ is an eigenvalue of the operator $A$ if and only if $k \gamma e^{\tau(a-b)} e^{-b}=1$.

In the rest of the paper, we shall assume that $k \gamma\left(e^{-a}-\right.$ $\left.e^{-b}\right) \neq a-b$ and $k \gamma e^{\tau(a-b)} e^{-b} \neq 1$. According to Theorem 2 and Lemma 3, we have the following result.

Theorem 4. If $k \gamma e^{\tau(a-b)} e^{-b} \neq 1$, then $\sigma(A)$ consists of eigenvalues with finite multiplicity and $\sigma(A)=\{\lambda \in \mathbb{C}$ : $\left.\Delta(\lambda)=k \gamma e^{-\lambda \tau}\left(\left(e^{-\lambda-a}-e^{-b}\right) /(\lambda+a-b)\right)-1=0\right\}$. of $A$.

In the sequel, we will further study the spectral properties

Lemma 5. Let $k \neq 0$ and $\sigma=k^{2} \gamma^{2} / 2-a$. There exists $M_{1}>0$ such that

$$
\begin{aligned}
\Sigma & =\left\{\lambda \in \mathbb{C} \mid \operatorname{Re} \lambda \leq \sigma, \operatorname{Im} \lambda \geq M_{1} e^{-2(1+\tau) \operatorname{Re} \lambda}\right\} \\
& \subseteq \rho(A) .
\end{aligned}
$$

Moreover, for $\lambda \in \Sigma$,

$$
\left|k \gamma e^{-\lambda \tau} \frac{e^{-b}-e^{-\lambda-a}}{\lambda+a-b}\right| \leq \frac{1}{2} .
$$

Proof. Let $\lambda=x+i y$ and let $x$ and $y$ be real numbers. Straightforward calculations show that

$$
\begin{gathered}
\left|k \gamma e^{-\lambda \tau} \frac{e^{-b}-e^{-\lambda-a}}{\lambda+a-b}\right|=|k| \gamma e^{-\tau x}\left|\frac{e^{-b}-e^{-x-a} e^{i y}}{x+a-b+i y}\right| \\
=|k| \gamma e^{-\tau x}\left|\frac{e^{-2 b}+e^{-2(x+a)}-2 e^{-(x+a+b)} \cos y}{(x+a-b)^{2}+y^{2}}\right|^{1 / 2} \\
=|k| \gamma\left|\frac{e^{2 x-2 b}+e^{-2 a}-2 e^{(x-a-b)} \cos y}{e^{2(\tau+1) x}(x+a-b)^{2}+e^{2(\tau+1) x} y^{2}}\right|^{1 / 2} .
\end{gathered}
$$

If $|y|>e^{-2(\tau+1) x}$, we have

$$
\begin{aligned}
& |k| \gamma\left|\frac{e^{2 x-2 b}+e^{-2 a}-2 e^{(x-a-b)} \cos y}{e^{2(\tau+1) x}(x+a-b)^{2}+e^{2(\tau+1) x} y^{2}}\right|^{1 / 2} \\
& \quad \leq|k| \gamma\left|\frac{e^{2 x-2 b}+e^{-2 a}+2 e^{(x-a-b)}}{e^{2(\tau+1) x}(x+a-b)^{2}+e^{-2(\tau+1) x}}\right|^{1 / 2} \longrightarrow 0
\end{aligned}
$$

as $x \rightarrow-\infty$. This means that there exists a constant $K_{1}>0$ such that

$$
\left|k \gamma e^{-\lambda \tau} \frac{e^{-b}-e^{-\lambda-a}}{\lambda+a-b}\right| \leq \frac{1}{2}
$$

$$
\text { if } \operatorname{Re} \lambda<-K_{1}, \operatorname{Im} \lambda \geq e^{-2(\tau+1) \operatorname{Re} \lambda} .
$$

Meanwhile, it follows from

$$
\left|k \gamma e^{-\lambda \tau} \frac{e^{-b}-e^{-\lambda-a}}{\lambda+a-b}\right| \leq|k| \gamma e^{-\tau x} \frac{e^{-b}+e^{-x-a}}{|y|}
$$

that $\left|k \gamma e^{-\lambda \tau}\left(\left(e^{-b}-e^{-\lambda-a}\right) /(\lambda+a-b)\right)\right|$ converges to 0 uniformly with respect to $-K_{1} \leq x \leq \sigma$ as $|y| \rightarrow \infty$. Thus, there exists a constant $K_{2}>0$ such that

$$
\left|k \gamma e^{-\lambda \tau} \frac{e^{-b}-e^{-\lambda-a}}{\lambda+a-b}\right| \leq \frac{1}{2}
$$

$$
\text { if }|\operatorname{Im} \lambda|>K_{2},-K_{1} \leq \operatorname{Re} \lambda \leq \sigma .
$$

Take $M_{1}>\max \left\{1, K_{2} e^{2(1+\tau) \sigma}\right\}$. It follows from (26) and (28) that

$$
\left|k \gamma e^{-\lambda \tau} \frac{e^{-b}-e^{-\lambda-a}}{\lambda+a-b}\right| \leq \frac{1}{2}, \quad \text { for } \lambda \in \Sigma
$$


Moreover, by Theorem 4, we have that $\Sigma \subset \rho(A)$. Thus the proof of the Lemma 5 is complete.

Now, we give the eventual regularity of the $C_{0}$-semigroup $(T(t))_{t>0}$ generated by the operator $A$.

Theorem 6. For any $k \neq 0$, there exists $t_{0}>0$ such that $C_{0}$-semigroup $(T(t))_{t \geq 0}$ generated by the operator $A$ is differentiable for $t>t_{0}$.

Proof. It follows from (23) that $|\Delta(\lambda)| \leq 1 / 2$ for $\lambda \in \Sigma$. Here, $\Delta(\lambda)$ is defined in the proof of Theorem 4 . Set

$$
\begin{aligned}
& \Sigma_{1}=\left\{\lambda \in \mathbb{C} \mid \operatorname{Re} \lambda \leq-a, \operatorname{Im} \lambda \geq M_{1} e^{-2(1+\tau) \operatorname{Re} \lambda}\right\}, \\
& \Sigma_{2} \\
& \quad=\left\{\lambda \in \mathbb{C} \mid-a \leq \operatorname{Re} \lambda \leq \sigma, \operatorname{Im} \lambda \geq M_{1} e^{-2(1+\tau) \operatorname{Re} \lambda}\right\} .
\end{aligned}
$$

It is easy to see that $\Sigma=\Sigma_{1} \cup \Sigma_{2}$. Moreover, it follows from (20) that

$$
\begin{aligned}
& R(\lambda, A)\left(\begin{array}{l}
h(x) \\
g(x)
\end{array}\right) \\
& =\left(\begin{array}{c}
k \gamma w(1) \int_{0}^{x} e^{-(\lambda+a)(x-s)} e^{-b s} d s+\int_{0}^{x} e^{-(\lambda+a)(x-s)} h(s) d s \\
w(0) e^{-\lambda \tau x}+\tau \int_{0}^{x} e^{-\lambda \tau(x-s)} g(s) d s
\end{array}\right),
\end{aligned}
$$

in which

$$
\begin{gathered}
w(0)=\frac{1}{\Delta(\lambda)}\left[\int_{0}^{1} e^{-(\lambda+a)(1-s)} h(s) d s\right. \\
\left.+\tau r(\lambda) \int_{0}^{1} e^{-\lambda \tau(1-s)} g(s) d s\right], \\
w(1)=\frac{1}{\Delta(\lambda)}\left[\tau \int_{0}^{1} e^{-\lambda \tau(1-s)} g(s) d s\right. \\
\left.+e^{\lambda \tau} \int_{0}^{1} e^{-(\lambda+a)(1-s)} h(s) d s\right]
\end{gathered}
$$

are given in (19) and $r(\lambda)=-k \gamma e^{-(\lambda+a)} \int_{0}^{1} e^{(\lambda+a-b) s} d s$. If we let $\|\cdot\|_{2}$ be the usual norm of $L^{2}(0,1)$, then we have

$$
\begin{aligned}
& |r(\lambda)| \leq \begin{cases}|k| \gamma e^{-\operatorname{Re} \lambda}, & \lambda \in \Sigma_{1} ; \\
|k| \gamma \int_{0}^{1} e^{\left(\omega_{0}+a-b\right) s} d s, & \lambda \in \Sigma_{2},\end{cases} \\
& \left|\int_{0}^{x} e^{-(\lambda+a)(x-s)} e^{-b s} d s\right| \leq \begin{cases}e^{-\operatorname{Re} \lambda}, & \lambda \in \Sigma_{1} ; \\
1, & \lambda \in \Sigma_{2},\end{cases} \\
& \left|\int_{0}^{x} e^{-(\lambda+a)(x-s)} h(s) d s\right| \leq \begin{cases}e^{-\operatorname{Re} \lambda}\|h\|_{2}, & \lambda \in \Sigma_{1} ; \\
\|h\|_{2}, & \lambda \in \Sigma_{2} ;\end{cases}
\end{aligned}
$$

$\forall x \in[0,1], h(x) \in L^{2}(0,1)$,

$$
\begin{aligned}
\left|\int_{0}^{x} e^{-\lambda \tau(x-s)} g(s) d s\right| \leq & \begin{array}{ll}
e^{-\tau \operatorname{Re} \lambda}\|g\|_{2}, & \lambda \in \Sigma_{1} \\
e^{a \tau}\|g\|_{2}, & \lambda \in \Sigma_{2}
\end{array} \\
& \forall x \in[0,1], g(x) \in L^{2}(0,1) .
\end{aligned}
$$

It follows from (33)-(36) that

$$
\begin{aligned}
& |w(0)| \leq \begin{cases}L_{1} e^{-(\tau+1) \operatorname{Re} \lambda}\left\|(h, g)^{\top}\right\|, & \lambda \in \Sigma_{1} ; \\
L_{2}\left\|(h, g)^{\top}\right\|, & \lambda \in \Sigma_{2} ;\end{cases} \\
& |w(1)| \leq \begin{cases}L_{1} e^{-(\tau+1) \operatorname{Re} \lambda}\left\|(h, g)^{\top}\right\|, & \lambda \in \Sigma_{1} ; \\
L_{2}\left\|(h, g)^{\top}\right\|, & \lambda \in \Sigma_{2},\end{cases}
\end{aligned}
$$

in which

$$
\begin{aligned}
& L_{1}=2 \sqrt{2} \max \{\tau, 1, \tau|k| \gamma\}, \\
& L_{2}=2 \sqrt{2} \max \left\{1, \tau e^{a \tau}, e^{\sigma \tau}, \tau|k| \gamma \int_{0}^{1} e^{(\sigma+a-b) s} d s\right\} .
\end{aligned}
$$

In light of estimates (35)-(38), we have

$$
\begin{aligned}
& \left\|R(\lambda, A)\left(\begin{array}{l}
h(x) \\
g(x)
\end{array}\right)\right\| \\
& \quad \leq \begin{cases}L_{3} e^{-2(\tau+1) \operatorname{Re} \lambda}\left\|(h, g)^{\top}\right\|, & \lambda \in \Sigma_{1} ; \\
L_{4}\left\|(h, g)^{\top}\right\|, & \lambda \in \Sigma_{2},\end{cases}
\end{aligned}
$$

in which

$$
\begin{aligned}
& L_{3}=2 \sqrt{\left(k \gamma L_{1}\right)^{2}+1+L_{1}^{2}+\tau^{2}}, \\
& L_{4}=2 \sqrt{\left(k \gamma L_{2}\right)^{2}+1+L_{2}^{2} e^{2 a \tau}+\tau^{2} e^{2 a \tau}} .
\end{aligned}
$$

Taking $L=\max \left\{L_{3}, L_{4} e^{2(1+\tau) \sigma}\right\}$, we have

$$
\begin{array}{r}
\left\|R(\lambda, A)\left(\begin{array}{c}
h(x) \\
g(x)
\end{array}\right)\right\| \leq L e^{-2(\tau+1) \operatorname{Re} \lambda}\left\|(h, g)^{\top}\right\|, \\
\forall\left(\begin{array}{l}
h(x) \\
g(x)
\end{array}\right) \in \mathscr{H}, \lambda \in \Sigma .
\end{array}
$$

Finally, by the definition of $\Sigma$ and (42), we have

$$
\|R(\lambda, A)\| \leq \frac{L}{M_{1}} M_{1} e^{-2(\tau+1) \operatorname{Re} \lambda} \leq \frac{L}{M_{1}} \operatorname{Im} \lambda, \quad \lambda \in \Sigma .
$$

It follows from Theorem 2.4.7 of [20] that there exists $t_{0}>0$ such that the $C_{0}$-semigroup $(T(t))_{t \geq 0}$ is differentiable for $t>$ $t_{0}$. The proof of Theorem 6 is complete.

Corollary 7. For any $k \neq 0$, there exists $t_{0}>0$ such that the $C_{0}$-semigroup $(T(t))_{t \geq 0}$ is compact for $t>t_{0}$. Moreover, the $C_{0^{-}}$ semigroup $(T(t))_{t \geq 0}$ satisfies the spectrum-determined growth condition. 
Proof. By Theorem 6, there exists $t_{0}>0$ such that the $C_{0^{-}}$ semigroup $(T(t))_{t \geq 0}$ is differentiable for $t>t_{0}$. Thus the $C_{0}$-semigroup $(T(t))_{t \geq 0}$ is norm continuous for $t>t_{0}$. In addition, we see from Theorem 2 that the operator $A$ has compact resolvent. Therefore, by Corollary 2.3.4 of [20], the $C_{0}$-semigroup $(T(t))_{t \geq 0}$ is compact for $t>t_{0}$.

\section{Exponential Stability Region of System (2)}

For convenience, we first state a feasible result of Ruan and Wei as follows (see [21, 22]).

Theorem 8. Consider the exponential polynomial

$$
\begin{aligned}
P( & \left.\lambda, e^{-\lambda \tau_{1}}, \ldots, e^{-\lambda \tau_{m}}\right) \\
= & \lambda^{n}+p_{1}^{(0)} \lambda^{n-1}+\cdots+p_{n-1}^{(0)} \lambda+p_{n}^{(0)} \\
& +\left[p_{1}^{(1)} \lambda^{n-1}+\cdots+p_{n-1}^{(1)} \lambda+p_{n}^{(1)}\right] e^{-\lambda \tau_{1}}+\cdots \\
& +\left[p_{1}^{(m)} \lambda^{n-1}+\cdots+p_{n-1}^{(m)} \lambda+p_{n}^{(m)}\right] e^{-\lambda \tau_{m}}
\end{aligned}
$$

where $\tau_{i} \geq 0(i=1,2, \ldots, m)$ and $p_{j}^{(i)}(i=0,1, \ldots, m-1, j=$ $1,2, \ldots, n)$ are constants. $A s\left(\tau_{1}, \tau_{2}, \ldots, \tau_{m}\right)$ vary, the sum of the orders of the zeros of $P\left(\lambda, e^{-\lambda \tau_{1}}, \ldots, e^{-\lambda \tau_{m}}\right)$ on the open right half-plane can change only if a zero appears on or crosses the imaginary axis.

In order to apply Theorem 8 to discuss the exponential stability region of system (2), we introduce the following result.

Theorem 9. When $\tau=0$ in (2), if $1-a+\ln (k \gamma)-\ln (3 \pi / 2)<0$, then system (2) is exponentially stable.

Proof. According to Theorem 3.2 of [5], we know that system (2) with $\tau=0$ is exponentially stable if and only if the spectral bound of the system operator is less than zero. However, Lemma 2.1 of [6] shows that the asymptotic expansions of the eigenvalues of the system operator are as follows:

$$
\begin{aligned}
\lambda_{n}= & -a+\ln (k \gamma)-\ln \left(\omega_{n}\right)+i\left(\omega_{n}-\frac{\ln \left(\omega_{n}\right)}{\omega_{n}}\right) \\
& +O\left(n^{-1}\right), \quad \omega_{n}=\left(2 n-\frac{1}{2}\right) \pi, n \geq 1 .
\end{aligned}
$$

It is easy to see that $\operatorname{Re}\left(\lambda_{n}\right) \leq \operatorname{Re}\left(\lambda_{n-1}\right)$ and the spectral bound of the system operator is less than $1-a+\ln (k \gamma)-\ln (3 \pi / 2)$. Thus, if $1-a+\ln (k \gamma)-\ln (3 \pi / 2)$ is less than zero, then system (2) with $\tau=0$ is exponentially stable. The proof of the Theorem is complete.

For further research, we introduce the following notations: $\alpha=k \gamma e^{-a}, \beta=k \gamma e^{-b}$, and $\eta=a-b$. Thus, $\lambda$ is a root of the characteristic equation $\Delta(\lambda)=0$ of the system operator (2) if and only if $\lambda$ is a root of the equation

$$
\lambda-\alpha e^{-\lambda(\tau+1)}-\beta e^{-\lambda \tau}+\eta=0,
$$

since $\lambda=-a+b$ is not the eigenvalue of the operator $A$ (see Lemma 3). Moreover, we have the following theorem.
Theorem 10. If $\alpha+\beta<\eta$ holds, then all roots of $\Delta(\lambda)=0$ have negative real parts.

Proof. Based on the observation above, it is sufficient to verify that all roots of (46) have negative real parts.

Firstly, it follows from Lemma 3 that $\lambda=0$ is not a root of (46). Moreover, it follows from Theorem 9 that all roots of (46) have negative real parts when $\tau=0$.

Now, we prove that (46) does not have root on imaginary axes. In fact, if $i \omega$ is a root of (46), then we have

$$
\begin{gathered}
i \omega-\alpha[\cos ((\tau+1) \omega)-i \sin ((\tau+1) \omega)] \\
-\beta[\cos (\tau \omega)-i \sin (\tau \omega)]+\eta=0
\end{gathered}
$$

Separating real and imaginary parts, we obtain

$$
\begin{aligned}
\eta=\alpha \cos ((\tau+1) \omega) & +\beta \cos (\tau \omega), \\
\omega & =-\alpha \sin ((\tau+1) \omega)-\beta \sin (\tau \omega) .
\end{aligned}
$$

This implies that

$$
\eta^{2}+\omega^{2}-\alpha^{2}-\beta^{2}=2 \alpha \beta \cos \omega
$$

which is equivalent to

$$
\frac{\omega^{2}+\eta^{2}-\alpha^{2}-\beta^{2}}{2 \alpha \beta}=\cos \omega,
$$

since $2 \alpha \beta$ is obviously not zero. The assumption $\alpha+\beta<$ $\eta$ implies that $\left(\eta^{2}-\alpha^{2}-\beta^{2}\right) / 2 \alpha \beta>1$. Hence, (50) is meaningless. This shows that (46) does not have imaginary root.

Finally, applying Theorem 8, we have that all roots of (46) have negative real parts for all $\tau>0$. The proof is completed.

Theorem 11. If $\alpha+\beta>\eta$ holds, then the equation $\Delta(\lambda)=0$ has at least one root with positive real parts for all $\tau>0$.

Proof. It is sufficient to verify that (46) has at least one root with positive real parts for all $\tau>0$.

Denote

$$
g(\lambda)=\lambda-\alpha e^{-\lambda(\tau+1)}-\beta e^{-\lambda \tau}+\eta \text {. }
$$

Then $g(0)=-\alpha-\beta+\eta<0$. Obviously, $\lim _{\lambda \rightarrow+\infty} g(\lambda)=$ $+\infty$ and hence there exists $\lambda_{0}>0$ such that $g\left(\lambda_{0}\right)=0$. This implies that $\lambda_{0}$ is a root of (46). This completes the proof.

In conclusion, we give the main result of the paper.

Theorem 12. If $k \gamma e^{\tau(a-b)} e^{-b} \neq 1,1-a+\ln (k \gamma)-\ln (3 \pi / 2)<0$, and $\alpha+\beta<\eta$ hold, then system (2) or (3) is exponentially stable for all $\tau>0$. However, if $k \gamma e^{\tau(a-b)} e^{-b} \neq 1$ and $\alpha+\beta>\eta$ hold, then system (2) or (3) is not stable.

Proof. According to Theorem 11, the second statement is obvious. Moreover, it follows from Theorems 4 and 10 that the spectral bound $s(A)$ of the operator $A$ is less than or equal to 
zero for all $\tau>0$. However, it is easy to see that the imaginary axis is not the asymptote of the zeros of the characteristic function $\Delta(\lambda)$ for all $\tau>0$. This means that the spectral bound $s(A)$ of the operator $A$ is less than zero for all $\tau>0$. Hence, Corollary 7 implies that the first statement holds. The proof is completed.

Remark 13. If we let $k \gamma=1$, then $1-a+\ln (k \gamma)-\ln (3 \pi / 2)<0$ is equivalent to $a>0$ and $\alpha+\beta<\eta$ is equivalent to $e^{-a}-$ $e^{-b}<a-b$. However, the mean value theorem implies that there exists $\xi \in(a, b)$ such that $e^{-a}-e^{-b}=-e^{-\xi}(a-b)$ and $e^{-a}-e^{-b}<a-b$ obviously holds if $a>b$. This means that if $a>b>0, k \gamma=1$, and $e^{\tau(a-b)} e^{-b} \neq 1$, system (2) or (3) is exponentially stable.

Remark 14. The above theorem gives a sufficient condition of the exponential stability of the monotubular heat exchanger equation with delay. This condition is applicable for all positive time delay $\tau$. This means that we answer the question in Section 1. We want to point out especially that the characteristic function $\Delta(\lambda)$ is the product of $e^{-\lambda \tau}$ and the characteristic function of the system without delay in the boundary observation. Moreover, The characteristic function $\Delta(\lambda)=0$, which is associated with one-order (both time and space) PDE with one delay, has the same type as that of the ODE with two delays. Thus, we can apply the result of [21] to discuss the distribution of roots of the characteristic equation $\Delta(\lambda)=0$, which is an important part of the paper.

\section{Conclusions and Further Research}

In this note, we give the stable regions of the monotubular heat exchanger equation with delay in boundary observation in light of the distribution of roots of the characteristic equation $\Delta(\lambda)=0$. More precisely, a sufficient condition of the exponential stability of the monotubular heat exchanger equation with delay is presented. In the further research, it is more realistic to discuss the effect of the external disturbance on the performance of the monotubular heat exchanger equation.

\section{Conflicts of Interest}

The authors declare that they have no conflicts of interest.

\section{Acknowledgments}

This work is supported by the National Natural Science Foundation of China under Grants 11201037, 11371071, and 11371070 and by the Natural Science Foundation of Liaoning Province under Grant 201602190.

\section{References}

[1] C. Z. Xu, J. P. Gauthier, and I. Kupka, "Exponential stability of the heat exchanger equation," in Proceedings of the Second European Control Conference, vol. 1, pp. 303-307, Groningen, The Netherlands, 1993.
[2] F. L. Huang, "Characteristic conditions for exponential stability of linear dynamical systems in Hilbert spaces," Ann. DiJerential Equations, vol. 1, pp. 43-56, 1985.

[3] J. Pruss, "On the spectrum of $C_{0}$-semigroups," Transactions of the American Mathematical Society, vol. 284, no. 2, pp. 847-857, 1984.

[4] H. Kanoh, "Control of heat exchangers by placement of closedloop poles," in Proceedings of the IFAC Symposium on Design Methods of Control Systems, pp. 662-667, Zurich, Switzerland, 1991.

[5] H. Sano, "Exponential stability of a mono-tubular heat exchanger equation with output feedback," Systems \& Control Letters, vol. 50, pp. 363-369, 2003.

[6] B.-Z. Guo and X.-Y. Liang, "Differentiability of the $C_{0^{-}}$ semigroup and failure of Riesz basis for a mono-tubular heat exchanger equation with output feedback: a case study," Semigroup Forum, vol. 69, no. 3, pp. 462-471, 2004.

[7] X. Yu and K. Liu, "Eventual regularity of the semigroup associated with the mono-tubular heat exchanger equation with output feedback," Systems \& Control Letters, vol. 55, pp. 859$862,2006$.

[8] I. Gumowski and C. Mira, Optimization in Control Theory and Practice, Cambridge University Press, Cambridge, 1968.

[9] R. Datko, "Not all feedback stabilized hyperbolic systems are robust with respect to small time delays in their feedbacks," SIAM Journal on Control and Optimization, vol. 26, no. 3, pp. 697-713, 1988.

[10] I. H. Suh and Z. Bien, "Use of Time-Delay Actions in the Controller Design," IEEE Transactions on Automatic Control, vol. 25, no. 3, pp. 600-603, 1980.

[11] W. H. Kwon, G. Won Lee, and S. Woo Kim, "Performance improvement using time delays in muhivariable controller design," International Journal of Control, vol. 52, no. 5, pp. 1455$1473,1990$.

[12] H. Logemann, R. Rebarber, and G. Weiss, "Conditions for robustness and nonrobustness of the stability of feedback systems with respect to small delays in the feedback loop," SIAM Journal on Control and Optimization, vol. 34, no. 2, pp. 572-600, 1996.

[13] B.-Z. Guo and K.-Y. Yang, "Dynamic stabilization of an Euler-Bernoulli beam equation with time delay in boundary observation," Automatica. A Journal of IFAC, the International Federation of Automatic Control, vol. 45, no. 6, pp. 1468-1475, 2009.

[14] B.-Z. Guo, C.-Z. Xu, and H. Hammouri, "Output feedback stabilization of a one-dimensional wave equation with an arbitrary time delay in boundary observation," ESAIM. Control, Optimisation and Calculus of Variations, vol. 18, no. 1, pp. 22-35, 2012.

[15] S. Nicaise and C. Pignotti, "Stability and instability results of the wave equation with a delay term in the boundary or internal feedbacks," SIAM Journal on Control and Optimization, vol. 45, no. 5, pp. 1561-1585, 2006.

[16] F. Zheng and L. Fu, "Spectral method for reliability analysis of a complex redundant system," ICIC Express Letters, Part B: Applications, vol. 4, no. 6, pp. 1535-1541, 2013.

[17] G. Q. Xu, S. P. Yung, and L. K. Li, "Stabilization of wave systems with input delay in the boundary control," ESAIM. Control, Optimisation and Calculus of Variations, vol. 12, no. 4, pp. 770785, 2006. 
[18] F. Zheng, L. Fu, and M. Teng, "Exponential stability of a linear distributed parameter bioprocess with input delay in boundary control," Journal of Function Spaces and Applications, vol. 2013, Article ID 274857, 2013.

[19] R. F. Curtain and H. J. Zwart, "An Introduction to Infinitedimensional Linear Systems Theory," in Texts in Applied Mathematics, vol. 21, Springer, New York, NY, USA, 1995.

[20] A. Pazy, Semigroups of Linear Operator and Applications to Partial Differential Equations, Springer, Berlin, Germany, 1983.

[21] S. G. Ruan and J. J. Wei, "On the zeros of a third degree exponential polynomial with applications to a delayed model for the control of testosterone secretion," Mathematical Medicine and Biology: A Journal of the IMA, vol. 18, pp. 41-52, 2001.

[22] X.-1. Li and J.-j. Wei, "Stability and bifurcation analysis in a system of four coupled neurons with multiple delays," Acta Mathematicae Applicatae Sinica. English Series, vol. 29, no. 2, pp. 425-448, 2013. 


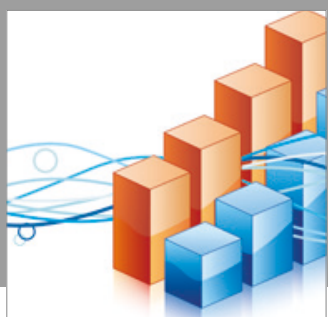

Advances in

Operations Research

vatersals

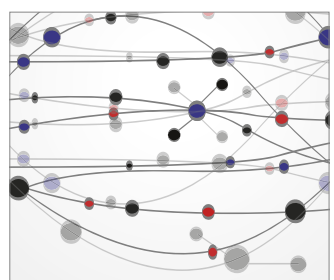

\section{The Scientific} World Journal
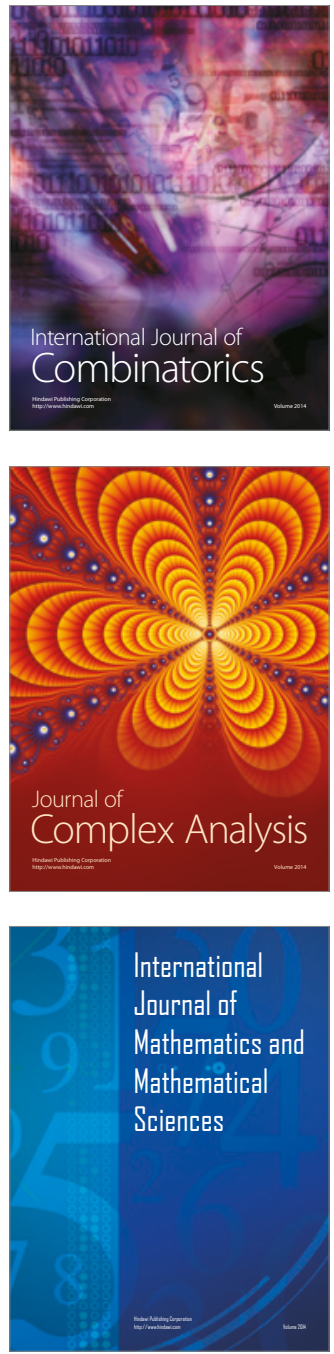
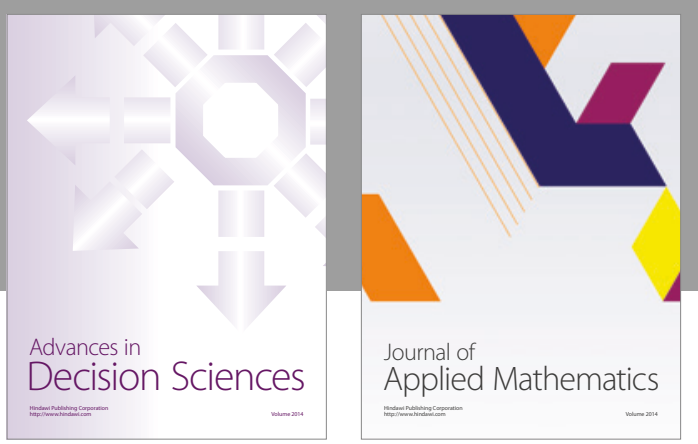

Algebra

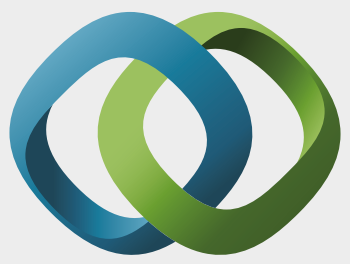

\section{Hindawi}

Submit your manuscripts at

https://www.hindawi.com
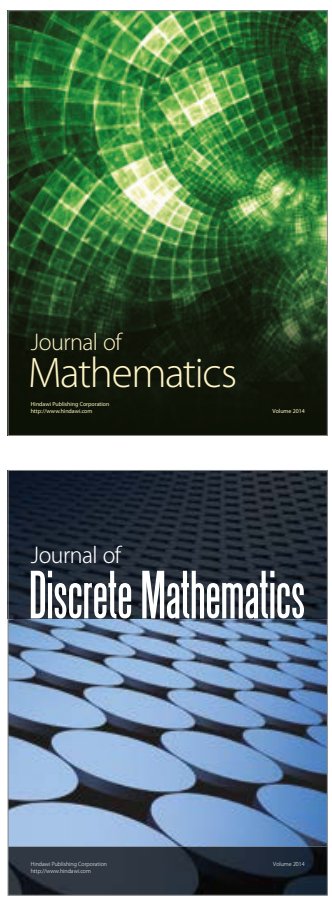

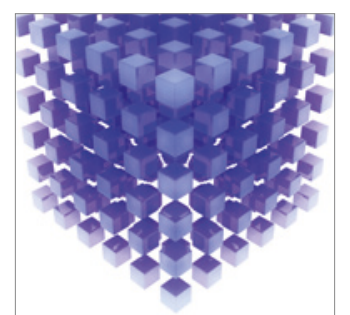

Mathematical Problems in Engineering
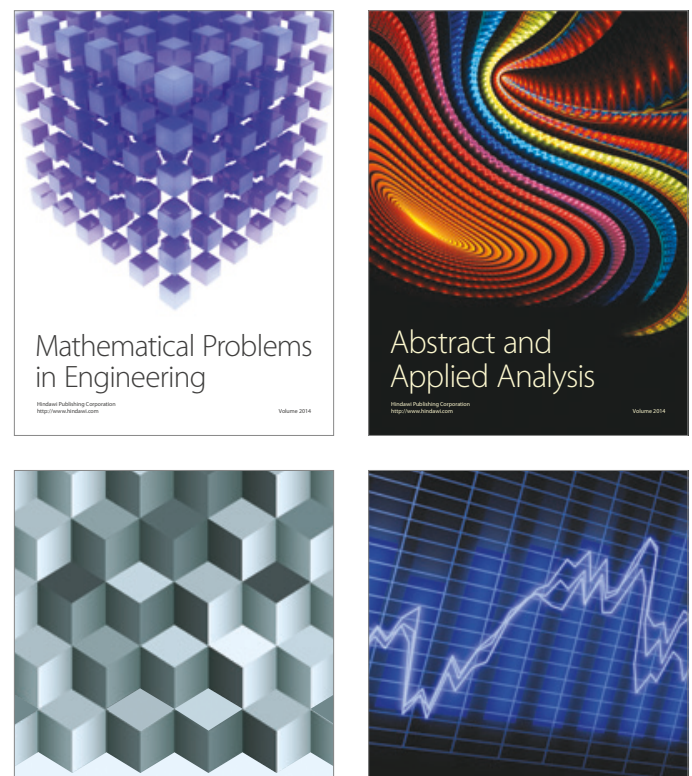

Journal of

Function Spaces

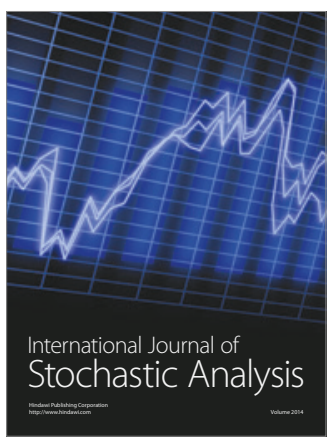

Probability and Statistics
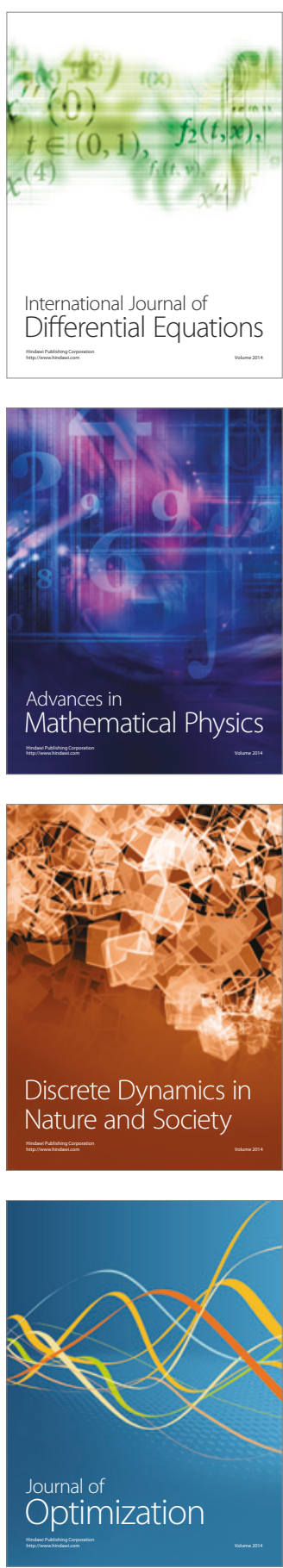